\title{
Serum Lactate Levels As a Predictor of Outcome in Pediatric Septic Shock
}

\author{
Dr.A.Umashankar MD (Paed $)^{1}$, Dr.Karthikeyan MD(Paed $)^{2}$, \\ ${ }^{1}$ Assistant Professor, Department of Paediatrics Coimbatore Medical College Hospital, Coimbatore \\ ${ }^{2}$ Assistant Professor, Department of Paediatrics Coimbatore Medical College Hospital, Coimbatore
}

\begin{abstract}
Introduction: Critical care medicine has remarkably improved in past few decades. Newer antibiotics are being introduced daily. Even then, sepsis is one of the leading causes of death worldwide. Infection which had cost millions of death in past are now being brought under control by effective immunization. Sepsis related deaths are more common in developing nations like India. Epidemiology of sepsis is changing. The reasons for this change are older infections previously fatal are now being eradicated due to effective antibiotics, immunization. Yet new infections are on the rise. Increased utilization of invasive devices and technology has lead to health care associated infections. Various biochemical indicators have been investigated to diagnose, prognosticate and to guide treatment in children with septic shock.

In physiological conditions lactate is not normally excreted by kidneys, because the renal threshold is approximately 5 to $6 \mathrm{mmol} / \mathrm{l}$. Renal cortex produce glucose from lactate by gluconeogenesis. When renal blood flow is compromised as in septic shock, clearance of lactate can be impaired causing elevated lactate levels.

Many pathological conditions of septic shock cause elevation of lactate level by increased production or impaired clearance. This findings make serum lactate - a potential biomarker in critically ill patients.

Sepsis and septic shock is one of the leading causes for admission in intensive care unit. Septic shock causes millions of children deaths every year. Mortality is high even with advanced intensive care . Studies have been conducted and new researches are ongoing to find biomarkers which will help in early identification, prognostication and treatment in critically ill children .This will help to reduce morbidity and mortality Serum lactate is one of the biomarkers which helps in predicting outcome in children with septic shock when measured serially.
\end{abstract}

Objective: To assess Serum lactate level as predictor of outcome in pediatric septic shock.

Secondary objectives: To determine other predictors of poor outcome in septic shock

Study Design: Nested case control study

Study Place: Pediatric intensive care unit, Coimbatore Medical College and Hospital

Study Period: December 2014 - September 2015

Study Population: 3 months -12 years children admitted in PICU with septic shock

Method: All Children admitted with septic shock in PICU between age 3 months to 12 years during study period was assessed for serum lactate.

Results: Out of 55 children, boy children were 30 , girl children were $2547.3 \%$ of children were under age of 1 year, $38.2 \%$ were between 1 and 5 years $.14 .5 \%$ o children were between 5 and 12 years Pneumonia $(65.5 \%)$ was the most common cause for septic shock

Anemia was observed in $69.1 \%$ of cases and undernutrition was observed in $72.7 \%$ of cases

Organ dysfunction other than cardiovascular dysfunction was observed in $36(65.5 \%)$ of children of which respiratory dysfunction was observed in 18 cases

Mortality in our study was $43.6 \%$ out of 55 children with septic shock, 31 were survivors and 24 children were non-survivors .

Mortality was highest in children 3 months -1 year $48 \%$ )

Among children with urosepsis , Escherichia coli was the causative organism

Both lactate at 0 to 3 hours and 24 hours lactate was significantly high in non-survivors

Apart from serum lactate, presence of organ dysfunction and under nutrition were significantly associated with poor outcome.

Initial serum lactate value $\geq 41 \mathrm{mg} / \mathrm{dl}(4.55 \mathrm{mmol} / \mathrm{L})$ and serum lactate value of $\geq 39.2 \mathrm{mg} / \mathrm{dl}(4.35 \mathrm{mmol} / \mathrm{L})$ at 24 hours were significant predictors of poor outcome in children with septic shock.

Conclusion: study demonstrated high serum lactate was associated with higher mortality .serial determination of serum lactate was a good predictor of poor outcome.

Early recognition of risk factors will help in timely appropriate therapy and thereby will help in reducing mortality and morbidity in pediatric septic shock.

Key words: Septic shock, Serum lactate 
Sample size:

\section{Methodology}

Sample size estimation formula, $Y=(\mu 1-\mu 2) /$

$\mathrm{Y}=$ difference between two population means

Population standard deviation

$\mathrm{Y}=0.56$

Corresponding to power at 0.50 ,estimated sample size is 49

Sampling technique:

Children admitted with septic shock on consecutive days between 7 am to 7 pm during the study period

\section{Statistical Analysis}

Was carried out using descriptive and inferential statistics. The chi square test was used to test the statistical significance of relationship between two variables. $T$ test was used to determine if there is a significant difference between the mean of two groups. The cut off value was estimated by using Receiver Operating Characteristics Curve analysis. Analysis reveals graphical relationship between sensitivity and 1specificity.odds ratio is a measure of association between exposure and outcome

Age Distribution Of Study Population

TABLE 1

\begin{tabular}{|l|l|l|}
\hline Age & Frequency & Percent \\
\hline 3 months -12 months & 26 & $47.3 \%$ \\
\hline 13 months -60 months & 21 & $38.2 \%$ \\
\hline 61 months -144 months & 8 & $14.5 \%$ \\
\hline Total & 55 & $100 \%$ \\
\hline
\end{tabular}

Chart 1

AGE

3 months - 12 months

13 months - 60 months

61 months - 144 months

$15 \%$

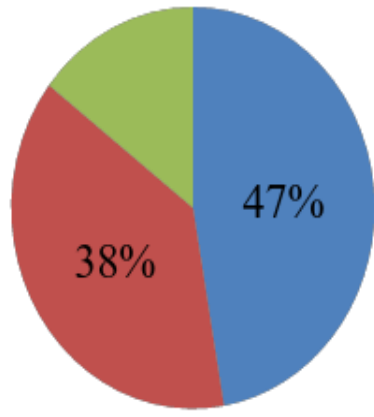

Gender

Table 2

\begin{tabular}{|l|l|l|}
\hline Sex & Frequency & Percent \\
\hline Male & 30 & $54.5 \%$ \\
\hline Female & 25 & $45.5 \%$ \\
\hline Total & 55 & $100 \%$ \\
\hline
\end{tabular}




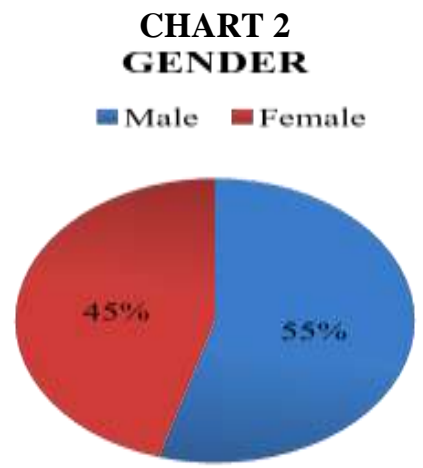

Diagonsis
Table 3
\begin{tabular}{|l|l|l|}
\hline Diagnosis & Frequency & Percent \\
\hline Urosepsis & 3 & $5.5 \%$ \\
\hline Bronchopneumonia & 36 & $65.5 \%$ \\
\hline Acute Diarrhoeal Disease & 8 & $14.5 \%$ \\
\hline Liver Abscess & 1 & $1.8 \%$ \\
\hline Meningitis & 7 & $12.7 \%$ \\
\hline Total & 55 & $100 \%$ \\
\hline
\end{tabular}

III. Discussion

The total number of children with septic shock admitted during the study period were 55 in number.Out of these 30 were boys and 25 were girls.

Anemia was observed in 38 cases. Undernutrition was observed in 40 cases. There were 31 survivors and 24 non survivors.mortality was $43.6 \%$. Bronchopneumonia $(65.5 \%)$ was the most cause of septic shock in our study followedby acute diarrhoeal disease(14.5\%),meningitis(12.7\%)urosepsis(5.5\%).Both initial and lactate at 24 hours were significantly high in non survivors $(\mathrm{p}<0.001)$. the association between lactate measured at 0 to 3 hours and outcome was significant.Also there was significant association between lactate measured at 24 hours and outcome. Initial lactate level of $>41 \mathrm{mg} / \mathrm{dl}$ had sensitivity of $87 \%$ and specificity of $95.8 \%$. . Lactate level at 24 hours had sensitivity of $90 \%$ and specificity of $95.8 \%$. High lactate level, presence of organ dysfunction ,and under nutrition were independently associated with poor outcome. In our study, initial lactate level of $\geq 41$ $\mathrm{mg} / \mathrm{dl}(4.55 \mathrm{mmol} / \mathrm{L})$ and 24 hours lactate level of $\geq 39.2 \mathrm{mg} / \mathrm{dl}(4.35 \mathrm{mmol} / \mathrm{L})$ was significantly associated with poor outcome . This is comparable to previous study conducted by Kana Ram Jat et al in which lactate $\geq 5$ $\mathrm{mmol} / \mathrm{L}$ or $45 \mathrm{mg} / \mathrm{dl}$ was significantly associated with poor outcome .In contrast to our study ,Duke et al reported lactate level of $\geq 3 \mathrm{mmol} / \mathrm{L}$ was a significant predictor of outcome .But in the study conducted by Duke et al, children with sepsis were the study population.Barat Ramakrishna et al studied children with pneumonia and reported a cut off of $>2 \mathrm{mmol} / \mathrm{l}$. Organ dysfunction other than cardiovascular dysfunction was observed in $36(65.5 \%$ ) cases.This is comparable to study by Kana Ram Jat et al in which organ dysfunction was observed in $66.7 \%$ of cases.Respiratory dysfunction(50\%) was the most common next to cardiovascular organ system dysfunction.

Age,sex and presence of anemia were not significantly different between non survivors and survivors.Presence of undernutrition and organ dysfunction were significantly associated with death.

\section{IV .Results}

Out of 55 children, boy children were 30 , girl children were $2547.3 \%$ of children were under age of 1 year,38.2\% were between 1 and 5 years $.14 .5 \%$ o children were between 5 and 12 years Pneumonia $(65.5 \%)$ was the most common cause for septic shock

Anemia was observed in $69.1 \%$ of cases and undernutrition was observed in $72.7 \%$ of cases

Organ dysfunction other than cardiovascular dysfunction was observed in $36(65.5 \%)$ of children of which respiratory dysfunction was observed in 18 cases

Mortality in our study was $43.6 \%$ out of 55 children with septic shock, 31 were survivors and 24 children were non-survivors .

Mortality was highest in children 3 months -1 year $(48 \%$ )

Among children with urosepsis, Escherichia coli was the causative organism

Both lactate at 0 to 3 hours and 24 hours lactate was significantly high in non-survivors

Apart from serum lactate, presence of organ dysfunction and under nutrition were significantly associated with poor outcome. 
Initial serum lactate value $\geq 41 \mathrm{mg} / \mathrm{dl}(4.55 \mathrm{mmol} / \mathrm{L}$ ) and serum lactate value of $\geq 39.2 \mathrm{mg} / \mathrm{dl}(4.35 \mathrm{mmol} / \mathrm{L})$ at 24 hours were significant predictors of poor outcome in children with septic shock.

\section{Conclusion}

Septic shock is one of the most common cause for mortality and morbidity in children .Early recognition and goal directed therapy is necessary to prevent complications.

Our study demonstrated high serum lactate was associated with higher mortality .serial determination of serum lactate was a good predictor of poor outcome.

Early recognition of risk factors will help in timely appropriate therapy and thereby will help in reducing mortality and morbidity in pediatric septic shock

\section{References}

[1]. Koliski et al conducted a prospective observational study in 75 children admitted in intensive care unit for various illness

[2]. Kana ram Jat conducted a prospective observational study in 30 children admitted to PICU with septic shock .Their aim was to find correlate serum lactate level in children with septic shock with survival serum lactate level were measured in arterial blood 012 and at 24 hours after admission

[3]. A prospective cohort study was conducted by Scott et al in children younger than 19 years. Their objective was to test the utility of serum lactate testing in pediatric emergency department patients with system inflammatory response syndrome.

[4]. Zhoux et al retrospectively studied the relationship between blood lactate level and disease severity in 232 critically ill children

[5]. Duke et al conducted a prospective study among 31 children admitted with sepsis. Their objective was to assess the markers of perfusion which best discriminate survivors from non-survivors .

[6]. Garcia Sanz et al performed a prospective study in 500 consecutive children admitted to PICU .Their objective was to analyse and compare the prognostic value of blood lactate and pediatric index of mortality (PIM) score on admission to a PICU .

[7]. Hatherill et al conducted a prospective study to examine relationship between early hyperlactatemia , acidosis and mortality in children admitted to intensive care. 755 critically ill children were screened . 50 children with hyperlactatemia were studied .

[8]. Prospective study was conducted by Barat Ramakrishna et al to determine whether blood lactate measured at time of presentation to hospital predicted outcome in children with pneumonia in Malawi . the relative risk of death if lactate level above $2 \mathrm{mmol} / \mathrm{l}$ was 48 , sensitivity was $92 \%$, specificity $39 \%$ multivariate analysis showed that hypoxemia, hyperlactatemia, age $\leq 12$ month were independent risk factors for death from pneumonia.

[9]. Mark E.Mikkelson et al performed a retrospective cohort study in patients presenting to the emergency department with severe sepsis.

[10]. In a study performed by Hussain et al, lactate level was measured at admission and at 24 hrs . 137 surgical intensive care unit patients were studied. Initial and 24 hours lactate level was significantly elevated in non survivors versus survivors $(\mathrm{p}=0.002)$. Mortality also increased with increasing time for normalization of lactate. Mortality rate was $10 \%$ if lactate normalized within 24 hours , $24 \%$ for $>48$ hours and $67 \%$ if lactate did not return to normal.

[11]. Marecaux et al conducted a prospective observation study on 38 patients admitted with septic shock

[12]. Phua $\mathbf{J}$ et al compared the prognostic utility of lactate procalcitonin , amino terminal pro - B - type natriuretic peptide with each others and with cytokine like interleukin $-1 \beta$, interleukin -6 and TNF $\alpha$ levels and clinical severity scores and their prognostic utility in septic shock. 72 patients with septic shock were studied. The biomarkers were measured on first 3 days of stay in intensive care unit. Rising trend in lactate \& procalcitonin level between day 2 and 3 were better prognostic indicators. They concluded that serial lactate level will be useful for prognostication in septic shock. 